\title{
Recognition of facial expressions is influenced by emotional scene gist
}

\author{
RUTHGER RIGHARI \\ Tilburg University, Tilburg, The Netherlands \\ AND \\ BEATRICE DE GELDER \\ Tilburg University, Tilburg, The Netherlands \\ and Harvard Medical School, Charlestown, Massachusetts
}

\begin{abstract}
Recognition of facial expressions has traditionally been investigated by presenting facial expressions without any context information. However, we rarely encounter an isolated facial expression; usually, we perceive a person's facial reaction as part of the surrounding context. In the present study, we addressed the question of whether emotional scenes influence the explicit recognition of facial expressions. In three experiments, participants were required to categorize facial expressions (disgust, fear, happiness) that were shown against backgrounds of natural scenes with either a congruent or an incongruent emotional significance. A significant interaction was found between facial expressions and the emotional content of the scenes, showing a response advantage for facial expressions accompanied by congruent scenes. This advantage was robust against increasing task load. Taken together, the results show that the surrounding scene is an important factor in recognizing facial expressions.
\end{abstract}

We rarely encounter an isolated facial expression; usually, we perceive a person's facial reaction as part of the surrounding context (Barrett, Lindquist, \& Gendron, 2007; Frijda \& Tcherkassof, 1997). A great number of studies have investigated perception and recognition of facial expressions (Adolphs, 2002; Ekman, 1992), but only recently have researchers started to investigate the role of context information (Biederman, 1972) and whether this context (emotional scene gist) may influence the processing of facial expressions (Barrett et al., 2007; Carroll \& Russell, 1996; de Gelder et al., 2006; Frijda \& Tcherkassof, 1997; Righart \& de Gelder, 2006).

Although common sense suggests that knowing the scene context would improve the recognition of facial expressions, the literature is less clear about this issue and allows for contradictory predictions. One possibility is that facial expressions have a processing advantage irrespective of the context in which they are perceived. For example, target processing is slowed by negative facial expressions, even when the target bears no direct relationship to the facial expression (Eastwood, Smilek, \& Merikle, 2003). Other studies suggest that this effect may be caused by an early capture of attention for negative facial expressions (Hansen \& Hansen, 1988; Öhman, Lundqvist, \& Esteves, 2001).

An alternative view holds that emotional contexts hamper face processing by diverting attention away from faces. This perspective is based on the finding that individuals prefer to attend toward emotional scenes (Calvo \& Lang, 2005), perhaps preferentially to fearful scenes
(Blanchette, 2006; Öhman, Flykt, \& Esteves, 2001; but see also Schimmack \& Derryberry, 2005). For example, when observers have to decide on the orientation of a bar, target processing is slowed when the target is placed within a fearful scene (Erthal et al., 2005).

A third possibility is that the recognition of facial expressions is influenced by concurrently presented emotional information. In previous work, it has been shown that the judgment of a facial expression is influenced by the emotional information that precedes the facial expression (Carroll \& Russell, 1996; see also Kim et al., 2004), but this has not been shown for faces and emotional scene contexts that are presented simultaneously. Faster categorization for facial expressions has, however, been observed when the target of processing and the context are emotionally congruent (for emotional voices, see de Gelder et al., 2006; for emotional words, see De Houwer \& Hermans, 1994; for emotional bodies, see Meeren, van Heijnsbergen, \& de Gelder, 2005; for schematic facial expressions, see Fenske \& Eastwood, 2003). Event-related potential (ERP) studies suggest that bodily expressions (Meeren et al., 2005) and emotional scenes (Righart \& de Gelder, 2006) exert an early influence on the processing of facial expressions.

In the present study, we addressed the question of whether emotional scenes affect how facial expressions are recognized behaviorally, by measuring error rates and response times (RTs); this had not been investigated in our previous ERP study, because a task that was uncorrelated with emotion had been used (Righart \& de Gelder,

B. de Gelder, b.degelder@uvt.nl 

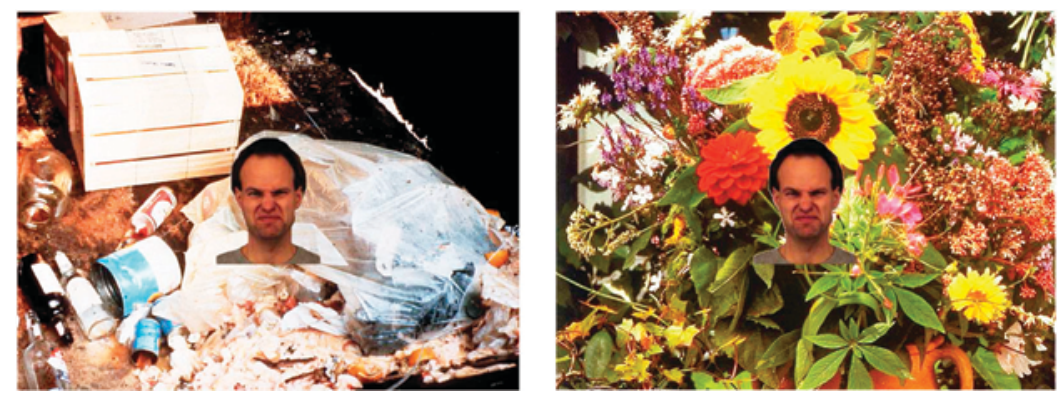

Figure 1. Example of a face-context stimulus. Facial expressions of fear, happiness, and disgust were paired with contexts of fear, happiness, and disgust. These pairs could constitute congruent emotions (left) - for instance, a facial expression of disgust in a context of garbage — or incongruent emotions (right) — the same expression, shown among flowers.

2006). In contrast with our previous study that had used only fearful facial expressions, in the present study, we used facial expressions of disgust, fear, and happiness that could form clear congruent and incongruent combinations with the accompanying emotional scenes.

Neuroimaging studies suggest that the brain processes emotions of fear and disgust by relatively separate neural systems. The amygdala has an important role in processing fear, whereas the insula-basal ganglia system has an important role in processing emotions of disgust (Calder, Lawrence, \& Young, 2001). It has often been argued that these systems respond, on an early stage of processing, to emotions, in order to be able to react to salient situations rapidly. If the facial expression and the accompanying emotional contexts are congruent, activations in these neural systems may increase during encoding. This may result in better and faster categorization of facial expressions.

Participants categorized facial expressions (disgust, fear, happiness) that were presented in task-irrelevant emotional scenes (disgusting, fearful, happy). We hypothesized that categorization of facial expressions would benefit more from congruent emotional contexts than from incongruent contexts. In Experiments 1A and 1B, we tested categorization of facial expressions in contexts using different presentation times and response alternatives. We tested different response alternatives because the combination of emotions might have an effect on the results (Tanaka-Matsumi, Attivissimo, Nelson, \& D'Urso, 1995). In Experiment 2, we tested the effect of increasing task load by introducing a concurrent task.

\section{EXPERIMENTS 1A AND 1B}

\section{Method}

Participants. The participants were 22 neurologically healthy undergraduate students ( 13 females; overall age $M=20.0$ years) from Tilburg University, who participated for course credit. Three were left-handed. All had normal or corrected-to-normal vision and provided informed consent. The study was performed in accordance with the ethical standards set in the 1964 Declaration of Helsinki.

Materials and Procedure. Face stimuli consisted of 36 color face photographs balanced for gender, taken from the Karolinska Directed Emotional Faces set (Lundqvist, Flykt, \& Öhman, 1998). Facial expressions were happy (12), disgusted (12), and fearful (12).
Contexts were 36 pictures of happy (12), disgusting (12), and fearful (12) scenes taken from the International Affective Picture System (Lang, Bradley, \& Cuthbert, 1999) and selected from the Web. In addition to the experiment, facial expressions and scenes were evaluated separately on the emotions of fear, disgust, and happiness. We selected contrasting emotions in order to introduce strong polarity between scenes, because the relative distance between emotional values of the stimuli might have an effect on categorization (TanakaMatsumi et al., 1995). Isolated facial expressions and neutral scenes were not used here. A problem of selecting a baseline context is that it is unclear whether neutral contexts are truly nonemotional.

Since the task consisted of categorizing facial expressions, participants were required to focus centrally on the face-scene stimulus. Faces were positioned centrally on emotional scenes (Figure 1) in order to avoid saccades. Faces and emotional scenes were carefully edited so that face pictures would not occlude critical parts of the scenes. The height and width of the faces and scenes was $9.0 \times 7.0 \mathrm{~cm}$ $\left(6.4^{\circ} \times 5.0^{\circ}\right)$ and $26.0 \times 35.0 \mathrm{~cm}\left(18.5^{\circ} \times 24.7^{\circ}\right)$, respectively.

Stimuli were presented for $200 \mathrm{msec}$. Participants used three buttons to categorize facial expressions (fearful, disgusted, happy),

Table 1

$t$ Test Planned Comparisons for Error Rates

\begin{tabular}{|c|c|c|c|c|}
\hline $\begin{array}{c}\text { Facial } \\
\text { Expression }\end{array}$ & Context & $t$ Value & $p$ & $d$ \\
\hline \multicolumn{5}{|c|}{ Experiment $1 \mathrm{~A}$} \\
\hline Disgust & $\begin{array}{l}\text { Disgusting-fearful } \\
\text { Disgusting-happy }\end{array}$ & $\begin{array}{l}-3.06 \\
-0.62\end{array}$ & $\begin{array}{l}<.01 \\
>.05\end{array}$ & $\begin{array}{l}-0.89 \\
-0.14\end{array}$ \\
\hline Fear & $\begin{array}{l}\text { Fearful-disgusting } \\
\text { Fearful-happy }\end{array}$ & $\begin{array}{l}-4.03 \\
-0.98\end{array}$ & $\begin{array}{l}<.001 \\
>.05\end{array}$ & $\begin{array}{l}-0.56 \\
-0.10\end{array}$ \\
\hline Happiness & $\begin{array}{l}\text { Happy-disgusting } \\
\text { Happy-fearful }\end{array}$ & $\begin{array}{l}-1.38 \\
-1.99\end{array}$ & $\begin{array}{l}>.05 \\
<.05\end{array}$ & $\begin{array}{l}-0.34 \\
-0.49\end{array}$ \\
\hline \multicolumn{5}{|c|}{ Experiment 1B } \\
\hline Disgust & $\begin{array}{l}\text { Disgusting-fearful } \\
\text { Disgusting-happy }\end{array}$ & $\begin{array}{l}-1.19 \\
-0.64\end{array}$ & $\begin{array}{l}>.05 \\
>.05\end{array}$ & $\begin{array}{l}-0.36 \\
-0.21\end{array}$ \\
\hline Fear & $\begin{array}{l}\text { Fearful-disgusting } \\
\text { Fearful-happy }\end{array}$ & $\begin{array}{l}-0.15 \\
-1.89\end{array}$ & $\begin{array}{l}>.05 \\
<.05\end{array}$ & $\begin{array}{l}-0.04 \\
-0.60\end{array}$ \\
\hline Happiness & $\begin{array}{l}\text { Happy-disgusting } \\
\text { Happy-fearful }\end{array}$ & $\begin{array}{r}0.93 \\
-0.95\end{array}$ & $\begin{array}{l}>.05 \\
>.05\end{array}$ & $\begin{array}{r}0.20 \\
-0.24\end{array}$ \\
\hline \multicolumn{5}{|c|}{ Experiment 2} \\
\hline Disgust & $\begin{array}{l}\text { Disgusting-fearful } \\
\text { Disgusting-happy }\end{array}$ & $\begin{array}{l}-3.06 \\
-2.62\end{array}$ & $\begin{array}{l}<.01 \\
<.05\end{array}$ & $\begin{array}{l}-0.42 \\
-0.20\end{array}$ \\
\hline Fear & $\begin{array}{l}\text { Fearful-disgusting } \\
\text { Fearful-happy }\end{array}$ & $\begin{array}{l}-1.60 \\
-1.19\end{array}$ & $\begin{array}{l}>.05 \\
>.05\end{array}$ & $\begin{array}{l}-0.27 \\
-0.19\end{array}$ \\
\hline Happiness & $\begin{array}{l}\text { Happy-disgusting } \\
\text { Happy-fearful }\end{array}$ & $\begin{array}{l}-0.96 \\
-1.15\end{array}$ & $\begin{array}{l}>.05 \\
>.05\end{array}$ & $\begin{array}{l}-0.25 \\
-0.35\end{array}$ \\
\hline
\end{tabular}


Table 2

$t$ Test Planned Comparisons for Response Times

\begin{tabular}{|c|c|c|c|c|}
\hline $\begin{array}{c}\text { Facial } \\
\text { Expression }\end{array}$ & Context & $t$ Value & $p$ & $d$ \\
\hline \multicolumn{5}{|c|}{ Experiment $1 \mathrm{~A}$} \\
\hline Disgust & $\begin{array}{l}\text { Disgusting-fearful } \\
\text { Disgusting-happy }\end{array}$ & $\begin{array}{l}-3.58 \\
-2.13\end{array}$ & $\begin{array}{l}<.001 \\
<.05\end{array}$ & $\begin{array}{l}-0.37 \\
-0.23\end{array}$ \\
\hline Fear & $\begin{array}{l}\text { Fearful-disgusting } \\
\text { Fearful-happy }\end{array}$ & $\begin{array}{l}-5.30 \\
-0.92\end{array}$ & $\begin{array}{l}<.001 \\
>.05\end{array}$ & $\begin{array}{l}-0.35 \\
-0.10\end{array}$ \\
\hline Happy & $\begin{array}{l}\text { Happy-disgusting } \\
\text { Happy-fearful }\end{array}$ & $\begin{array}{l}-5.58 \\
-3.30\end{array}$ & $\begin{array}{l}<.001 \\
<.01\end{array}$ & $\begin{array}{l}-0.33 \\
-0.23\end{array}$ \\
\hline \multicolumn{5}{|c|}{ Experiment 1B } \\
\hline Disgust & $\begin{array}{l}\text { Disgusting-fearful } \\
\text { Disgusting-happy }\end{array}$ & $\begin{array}{l}-0.36 \\
-1.37\end{array}$ & $\begin{array}{l}>.05 \\
>.05\end{array}$ & $\begin{array}{l}-0.06 \\
-0.20\end{array}$ \\
\hline Fear & $\begin{array}{l}\text { Fearful-disgusting } \\
\text { Fearful-happy }\end{array}$ & $\begin{array}{l}-2.01 \\
-1.61\end{array}$ & $\begin{array}{l}<.05 \\
>.05\end{array}$ & $\begin{array}{l}-0.30 \\
-0.16\end{array}$ \\
\hline Happy & $\begin{array}{l}\text { Happy-disgusting } \\
\text { Happy-fearful }\end{array}$ & $\begin{array}{l}-2.66 \\
-2.89\end{array}$ & $\begin{array}{l}<.01 \\
<.01\end{array}$ & $\begin{array}{l}-0.42 \\
-0.32\end{array}$ \\
\hline \multicolumn{5}{|c|}{ Experiment 2} \\
\hline Disgust & $\begin{array}{l}\text { Disgusting-fearful } \\
\text { Disgusting-happy }\end{array}$ & $\begin{array}{l}-2.73 \\
-2.49\end{array}$ & $\begin{array}{l}<.01 \\
<.05\end{array}$ & $\begin{array}{l}-0.21 \\
-0.13\end{array}$ \\
\hline Fearful & $\begin{array}{l}\text { Fearful-disgusting } \\
\text { Fearful--happy }\end{array}$ & $\begin{array}{l}-2.82 \\
-0.79\end{array}$ & $\begin{array}{l}<.01 \\
>.05\end{array}$ & $\begin{array}{l}-0.22 \\
-0.09\end{array}$ \\
\hline Happy & $\begin{array}{l}\text { Happy-disgusting } \\
\text { Happy-fearful }\end{array}$ & $\begin{array}{l}-1.89 \\
-1.95\end{array}$ & $\begin{array}{l}<.05 \\
<.05\end{array}$ & $\begin{array}{l}-0.23 \\
-0.18\end{array}$ \\
\hline
\end{tabular}

while ignoring the surrounding scene. Response mapping was counterbalanced across participants. Participants were instructed to respond using the index finger. RTs were recorded from stimulus onset. Responses were initiated from a keypad in order to control distance to the response buttons; this was monitored using a video system.

The experiment comprised eight separate blocks ( 72 trials), each block consisting of all possible emotion combinations of face and context, resulting in congruent pairs (FF, DD, HH, each 96 trials) and incongruent pairs (FD, FH; DF, DH; HD, HF; each 48 trials). The number of trials was equalized for congruent and incongruent trials to avoid predictability. The order of blocks was counterbalanced across participants.

Analyses. Error rates (percentage of incorrect responses) and RTs (averaged for correct responses) were analyzed for all conditions. Participants were removed from analyses if they were unable to classify more than 8 of 12 emotions for any of the three emotion categories.

RTs more than 2.5 standard deviations from the mean of each condition were removed from analysis for each participant separately, leading to less than $2.6 \%$ of the trials being removed. The mean and standard error of the mean are reported.

Main effects and interactions for facial expression (disgust, fear, happiness) and scene (disgusting, fearful, happy) were tested by a repeated measures ANOVA ( $\alpha=.05$, one-tailed). $p$ values were corrected by Greenhouse-Geisser epsilon, if appropriate. Partial eta squared $\left(\eta_{\mathrm{p}}^{2}\right)$ is reported as an estimate of effect size. In Tables 1 and 2, the $t$-test planned comparisons used to test our hypothesis of congruency effects are reported. In addition, Cohen's $d$ is reported and is defined as the difference between two means, divided by the pooled standard deviations for those means (Cohen, 1988). In Tables 3 and 4, the error rates and RTs are reported.

\section{Results}

Experiment 1A. Two participants were removed from analyses because they failed to classify more than 8 of 12 facial expressions correctly.

Error rates for judging facial expressions were, on average, $8.3 \%$. A main effect was found for facial expres- sion $\left[F(2,38)=29.45, p<.001, \eta_{\mathrm{p}}^{2}=.61\right]$. Error rates differed significantly across facial expressions of fear, disgust, and happiness: $t(19)=3.82, p<.01, d=1.29$ for fear-disgust; $t(19)=5.61, p<.001, d=1.65$ for disgust-happiness; and $t(19)=7.20, p<.001, d=2.06$ for fear-happiness. A main effect was also found for scene $\left[F(1.67,31.68)=7.38, p<.01, \eta_{\mathrm{p}}^{2}=.28\right]$, since more errors were made for faces in disgusting scenes and fearful scenes than for faces in happy scenes: $t(19)=4.30, p<$ $.001, d=0.37$ for disgust-happiness; $t(19)=2.54, p<$ $.05, d=0.42$ for fear-happiness.

The main effects were modified by an interaction $\left[F(4,76)=10.78, p<.001, \eta_{\mathrm{p}}^{2}=.36\right]$ for which the $t$-test comparisons are mentioned in Table 1. Fewer error responses were committed for disgusted facial expressions

Table 3

Mean Percentage Error Rates (PE) and Standard Errors (SEM) for Interaction Effects Between Facial Expressions and Contexts

\begin{tabular}{|c|c|c|}
\hline \multicolumn{3}{|c|}{ Context } \\
\hline Disgusting & Fearful & Happy \\
\hline PE $\quad S E M$ & SEM & SEM \\
\hline
\end{tabular}

Experiment 1A

\begin{tabular}{|c|c|c|c|c|c|c|}
\hline \multicolumn{7}{|l|}{ Main Effect } \\
\hline Facial expression & 6.6 & 0.9 & 17.2 & 2.4 & 1.0 & 0.5 \\
\hline Scene & 8.9 & 1.0 & 8.7 & 0.9 & 7.2 & 0.9 \\
\hline \multicolumn{7}{|l|}{ Interaction } \\
\hline Disgust & 4.6 & 0.8 & 9.9 & 1.7 & 5.2 & 1.0 \\
\hline Fear & 20.9 & 2.6 & 14.7 & 2.4 & 15.8 & 2.6 \\
\hline Happiness & 1.2 & 0.7 & 1.5 & 0.6 & 0.4 & 0.2 \\
\hline \multicolumn{7}{|c|}{ Experiment 2} \\
\hline \multicolumn{7}{|l|}{ Main Effect } \\
\hline Facial expression & 8.3 & 2.3 & 10.4 & 2.7 & 1.0 & 0.4 \\
\hline Scene & 6.4 & 1.3 & 6.8 & 1.3 & 6.5 & 1.2 \\
\hline \multicolumn{7}{|l|}{ Interaction } \\
\hline Disgust & 6.5 & 1.9 & 10.3 & 2.8 & 8.0 & 2.2 \\
\hline Fear & 11.8 & 3.3 & 8.9 & 2.4 & 10.7 & 2.7 \\
\hline Happiness & 1.0 & 0.5 & 1.3 & 0.7 & 0.7 & 0.2 \\
\hline
\end{tabular}

Table 4

Mean Response Times (RTs, in Milliseconds) and Standard Errors (SEM) for Interaction Effects Between Facial Expressions and Contexts

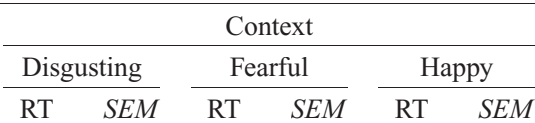

Experiment 1A

\begin{tabular}{|c|c|c|c|c|c|c|}
\hline Main Effect & & & & & & \\
\hline Facial expression & 953 & 26 & 1,061 & 35 & 803 & 22 \\
\hline Scene & 947 & 25 & 939 & 25 & 932 & 24 \\
\hline Interaction & & & & & & \\
\hline Disgust & 929 & 26 & 972 & 26 & 957 & 28 \\
\hline Fear & 1,094 & 35 & 1,037 & 38 & 1,053 & 34 \\
\hline Happiness & 818 & 23 & 807 & 21 & 784 & 23 \\
\hline & & perim & & & & \\
\hline Main Effect & & & & & & \\
\hline Facial expression & 1,634 & 106 & 1,637 & 65 & 1,242 & 67 \\
\hline Scene & 1,506 & 68 & 1,513 & 74 & 1,493 & 71 \\
\hline Interaction & & & & & & \\
\hline Disgust & 1,589 & 101 & 1,676 & 119 & 1,637 & 100 \\
\hline Fear & 1,664 & 63 & 1,611 & 68 & 1,634 & 70 \\
\hline Happiness & 1,266 & 72 & 1,253 & 68 & 1,208 & 64 \\
\hline
\end{tabular}


in disgusting and happy scenes than in fearful scenes. Fewer error responses were made for fearful facial expressions in fearful and happy scenes than in disgusting scenes. Fewer error responses were committed for happy facial expressions in happy scenes than in fearful scenes.

Analyses of the error responses for fearful facial expressions showed that a large proportion of errors was attributed to responding disgust (19.1\%), as compared with responding happy $(1.9 \%)[t(19)=6.67, p<.001]$, when fearful facial expressions were presented in disgusting contexts. This percentage was also higher than that attributed to responding disgust to fearful faces in fearful contexts $(12.8 \%)[t(19)=3.78, p<.001]$ and in happy contexts $(13.3 \%)[t(19)=4.82, p<.001]$.

Analyses of the error responses for disgusted facial expressions showed that a large proportion of errors was attributed to responding fear (9.7\%), as compared with responding happy $(0.2 \%)[t(19)=5.41, p<.001]$, when disgusted facial expressions were presented in fearful contexts. This percentage was also higher than the proportion of errors attributed to responding fear to disgusted facial expressions in disgusting contexts $(4.1 \%)[t(19)=3.38, p<.01]$ and in happy contexts $(4.0 \%)[t(19)=3.98, p<.001]$. The percentage of error responses for happy faces was low in all conditions $(<2 \%)$, and was therefore not analyzed.

The analysis of RTs showed a main effect for facial expression $\left[F(2,38)=61.56, p<.001, \eta_{\mathrm{p}}^{2}=.76\right]$ and scene $\left[F(2,38)=3.78, p<.05, \eta_{\mathrm{p}}^{2}=.17\right]$. Responses were fastest for facial expressions of happiness, intermediate for disgust, and slowest for fear $[t(19)=7.14, p<.001, d=$ 1.40 for happiness-disgust; $t(19)=9.95, p<.001, d=$ 0.97 for happiness-fear; and $t(19)=4.72, p<.001, d=$ 0.79 for disgust-fear]. Responses were faster for faces in happy scenes than for faces in disgusting scenes $[t(19)=$ $3.32, p<.01, d=0.14]$, but were not significantly different from responses for faces in fearful scenes $[t(19)=$ $1.58, p=.13$ for disgust-fear; and $t(19)=1.03, p=.32$ for happiness-fear].

The main effects were qualified by a significant interaction $\left[F(2.28,43.39)=7.47, p<.01, \eta_{\mathrm{p}}^{2}=.28\right]$ (see Figure 2A), for which the $t$-test planned comparisons are mentioned in Table 2. Comparisons between scenes showed that responses to facial expressions of disgust were faster if they were accompanied by disgusting, rather than happy and fearful, scenes. RTs were faster for fear facial expressions in fearful and happy scenes than for those in disgusting scenes. RTs for happy facial expressions were faster in happy scenes than in disgusting and fearful scenes.

Experiment 1B. In order to investigate whether the number of response alternatives and the emotion combinations would affect the results, we conducted another experiment to find whether blocks containing the different combinations of emotions would introduce different results. Previous studies have shown that the combination of emotions being tested may influence the results that are obtained (Tanaka-Matsumi et al., 1995); therefore, we hypothesized that the reliance on context information would produce different results in this experiment.

Another group of 15 participants $(M=21.8$ years $)$ were presented with face-scene stimuli in a two-alternative forced choice task in blocks with facial expressions of fear and disgust (FD block), fear and happiness (FH block), and disgust and happiness (DH block). Experiment 1A has shown that the context effects occur for short presentation times (see also Calvo \& Lang, 2005; Loftus, Nelson, \& Kallman, 1983). However, it is unknown how individuals categorize facial expressions when they are able to make several fixations on face and scene. The presentation time was therefore as long as participants needed for responding, with a maximum of $6,000 \mathrm{msec}$.

Our main interest was to test whether congruency effects would occur when participants have only two response alternatives. Analyses were broken down by block to test whether the combinations of emotions for each block would introduce different results. Therefore, main effects and interactions were analyzed for each block separately for facial expression and scene.

The error rates were, on average, less than $5 \%$. In the FD block, a significant main effect was found for facial expression $\left[F(1,14)=22.12, p<.001, \eta_{\mathrm{p}}^{2}=.61\right]$, because more errors were made for fearful faces $(M=$ $5.2 \%, S E M=0.7 \%)$ than for disgusted faces $(M=2.2 \%$, $S E M=0.5 \%)$. No significant main effects or interactions were found for the FH and HD blocks (see Table 1 for $t$-test planned comparisons).

The analyses for RTs showed interactions between facial expression and scene in the FD block $[F(1,14)=7.39, p<$ $.05, \eta_{\mathrm{p}}^{2}=.35$ ] (see Figure 2B), with RTs that were faster for fearful facial expressions in fearful $(M=838 \mathrm{msec}$, $S E M=36 \mathrm{msec})$ than in disgusting $(M=882 \mathrm{msec}$, $S E M=39 \mathrm{msec}$ ) scenes. The difference between disgusted facial expressions in disgusting scenes $(M=759 \mathrm{msec}$, $S E M=29 \mathrm{msec})$ and disgusted facial expressions in fearful scenes $(M=766 \mathrm{msec}, S E M=26 \mathrm{msec})$ did not attain significance (see Table 2 for $t$-test planned comparisons).

The FH experimental block showed an interaction between facial expression and scene $[F(1,14)=11.36, p<$ $\left..01, \eta_{\mathrm{p}}^{2}=.45\right]$; RTs to happy facial expressions were faster in happy $(M=590 \mathrm{msec}, S E M=22 \mathrm{msec})$ than in fearful $(M=617 \mathrm{msec}, S E M=23 \mathrm{msec})$ scenes, and RTs were not significantly faster for fearful facial expressions in fearful $(M=640 \mathrm{msec}, S E M=32 \mathrm{msec})$ than in happy $(M=658 \mathrm{msec}, S E M=28 \mathrm{msec})$ scenes.

The DH experimental block showed an interaction between facial expression and scene $[F(1,14)=11.01, p<$ $\left..01, \eta_{\mathrm{p}}^{2}=.44\right]$, showing that RTs were faster for happy facial expressions in happy $(M=600 \mathrm{msec}, S E M=$ $17 \mathrm{msec})$ than in disgusting $(M=634 \mathrm{msec}, S E M=$ $24 \mathrm{msec}$ ) scenes, whereas the difference between disgusted facial expressions in disgusting scenes $(M=588 \mathrm{msec}$, $S E M=17 \mathrm{msec}$ ), compared with those in happy scenes ( $M=601 \mathrm{msec}, S E M=13 \mathrm{msec})$, was not significant.

\section{Discussion}

Experiment 1A shows faster recognition of facial expressions in congruent emotional scenes, which was paralleled by error rates, indicating that there were no speed-accuracy trade-offs. Since these effects were found with presentation times that only allowed single fixations (Loftus et al., 1983), this indicates that the effects rely on rapid global 

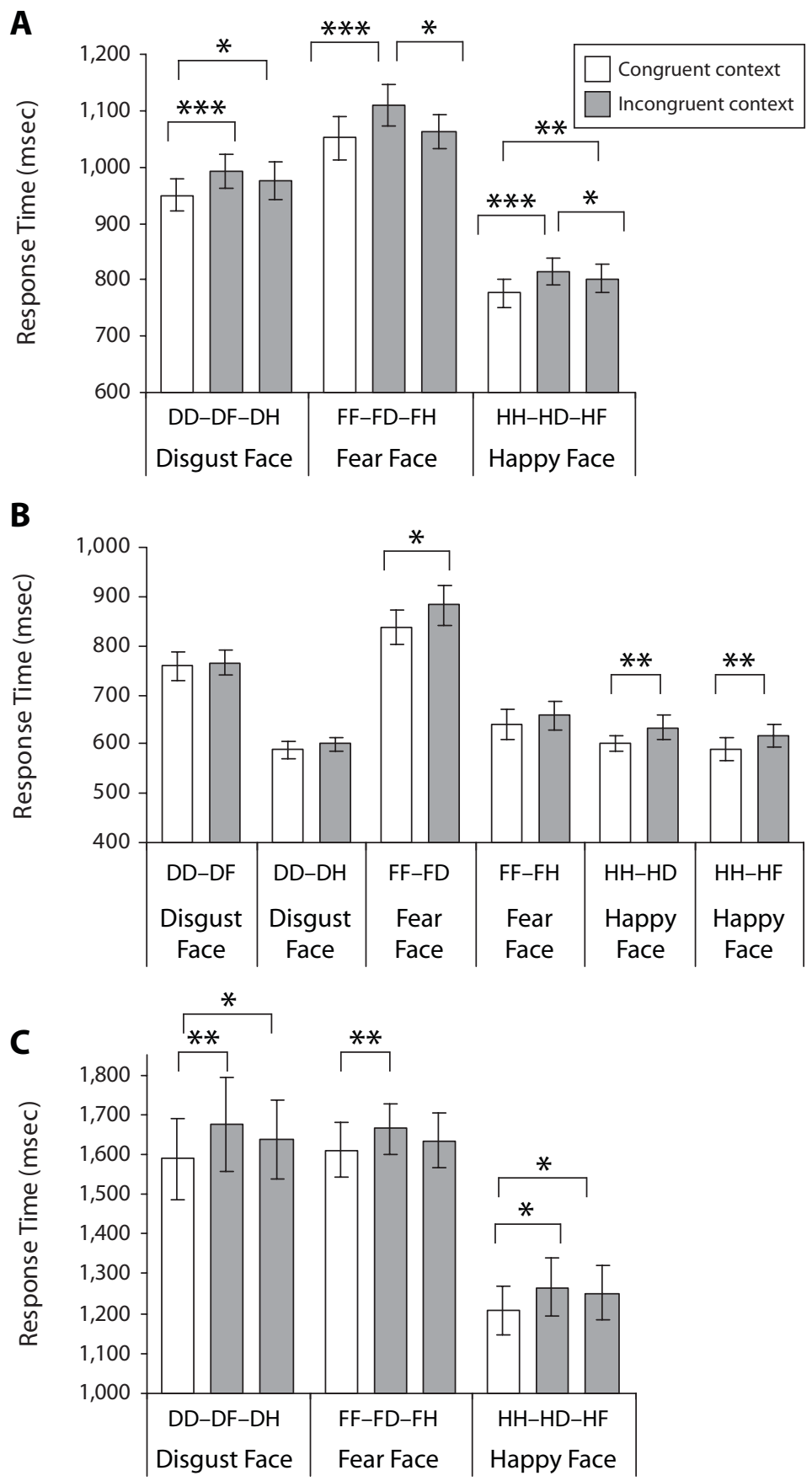

Figure 2. Mean response times for facial expressions in congruent (white bar) and incongruent (gray bar) contexts. (A) In Experiment 1A, a three-alternative choice task was used, in which participants categorized facial expressions of fear, disgust, and happiness. (B) In Experiment 1B, participants categorized these facial expressions in a two-alternative choice task. (C) In Experiment 2, task load was increased due to a distractor task. For the abbreviations, the first letter represents facial expression, and the second letter represents emotional scene: D, disgust; F, fear; H, happy. Error bars represent one standard error. ${ }^{*} p<.05 . \quad{ }^{* *} p<.01 . \quad{ }^{* * *} p<.001$. 
perceptual analysis of the scene. The emotional context influences the pattern of error responses, which shows that incorrect responses are biased toward the emotional context that accompanies the facial expression. This pattern was present for error responses to both fearful and disgusted facial expressions and is consistent with that found in an earlier study, in which an emotional story line that preceded facial expressions influenced the judgment of facial expressions in the direction of the emotional context (Carroll \& Russell, 1996). The present study shows that this result extends to experimental settings in which the facial expression and context are presented simultaneously. These effects were found for error responses and RTs for fear and disgust facial expressions, but were found only on RTs for happy facial expressions. Whereas the study by Carroll and Russell already showed that the judgment of facial expressions can be modified by a priori knowledge about a situation, the present data suggest that the judgment of facial expressions can be modified when individuals perceive this background information at the same time, as well. This suggests that context processing occurs rapidly and may directly modify our interpretations of what a facial expression means, which is obviously important in situations in which flexible adaptations are required.

Another important aspect is that the recognition of facial expressions of fear and happiness benefits from congruent scenes, but that this benefit was not found for facial expressions of disgust in Experiment 1B. The number of response alternatives may have contributed to the effect (see Tanaka-Matsumi et al., 1995). When individuals have to select among several alternatives, choices may rely more on the available context information, because facial expressions are more difficult to discriminate (Carroll \& Russell, 1996).

Another important result was that neither of the experiments showed significant effects for fearful facial expressions in fearful scenes, as compared with those for happy scenes. In Experiment 1B, there was not an effect for disgust facial expressions. However, the happy facial expressions showed congruency effects consistent with those shown in a study by Fenske and Eastwood (2003), who investigated the recognition of schematic target facial expressions that were flanked by congruent or incongruent schematic facial expressions.

The congruency effect, and overall RTs, as well, differed between fear and disgust. In the next experiment, we investigated whether the amount of attention dedicated to facial expressions of fear and disgust could explain the differential congruency effects that were observed. We investigated whether the task load, and thus the amount of attention dedicated to processing facial expressions, could explain the differences that were observed between facial expressions of fear and disgust.

\section{EXPERIMENT 2}

Irrelevant context information interferes with target processing only if the task load is relatively low (Lavie, 1995). If task load determines the degree to which context information is processed, then the effect of context on face processing may be canceled if the task load is high.

Similar to the effects that have been observed for nonemotional contexts (Lavie, 1995), emotional contexts interfere with target processing. Normally, task-irrelevant emotional information slows RTs. However, if the task load is increased, unpleasant scenes do not slow RTs any more (Erthal et al., 2005).

A concurrent task was used in order to investigate how the imposed task load influences the capacity for context processing. Besides the main task of categorizing facial expressions, participants were required to categorize a character that was overlaid on the face stimulus. If the concurrent task reduces attentional resources, it is expected that context effects will disappear. In contrast, if contexts affect face processing, irrespective of the amount of attention dedicated, RTs may still be faster for congruent pairs of facial expression and scene than for incongruent pairs.

\section{Method}

The experiment was identical to Experiment 1, except the additional task now required participants to categorize a character symbol displayed in the center of the face.

Participants. Sixteen neurologically healthy undergraduate students ( 8 females, overall mean age $=23$ years) were recruited from Tilburg University. Two participants were left-handed. All had normal or corrected-to-normal vision and provided informed consent.

Materials and Procedure. Similar face-scene pairs were used, except that an " $x$ " or "o" character was centered on each face. Characters were presented in small (10 points) or large (20 points) font size to avoid the possibility that features related to font size would cue the correct response. The overlap of the character on the face-scene stimulus was randomized in order to avoid a predictive relationship between characters and face-scene stimuli. The combination of face and character stimuli was counterbalanced across participants. Stimuli had a similar onset and were presented for $200 \mathrm{msec}$. Participants used three buttons for categorizing facial expressions (fear, disgust, happiness), after which they used two buttons to indicate the character that was presented.

Analyses. Analyses were identical to those in Experiment 1. We removed $2.4 \%$ of all trials as RT outliers.

\section{Results}

Two participants were removed from analyses: one because of an excessive number of misclassifications for fearful faces, and the other because of poor performance on the categorization task for characters ( $M=52 \%$ error). The remaining participants showed a relatively small percentage of errors on the character categorization task $(M=8.9 \%, S E M=1.3 \%)$.

The error rates for categorizing facial expressions in scenes were, on average, $6.6 \%$. A significant main effect was found for facial expression $[F(1.54,20.07)=6.14$, $\left.p<.01, \eta_{\mathrm{p}}^{2}=.32\right]$, in that more errors were made for fearful and disgusted than for happy facial expressions, since the comparisons of fear-happiness $[t(13)=3.61, p<.01$, $d=1.31]$ and disgust-happiness $[t(13)=3.28, p<.01$, $d=1.18]$ were significant. The main effect for scene was not significant.

However, a significant interaction was found between facial expression and scene $[F(1.70,22.10)=3.86, p<$ $\left..05, \eta_{\mathrm{p}}^{2}=.23\right]$. Fewer error responses were committed for 
disgust facial expressions in disgust than in fear and happy scenes. No significant effects of scene were found for facial expressions of fear and happy (see Table 1 for planned $t$-test comparisons).

Analyses for RTs showed a main effect for facial expression $\left[F(2,26)=20.63, p<.001, \eta_{\mathrm{p}}^{2}=.61\right]$, which was explained by RTs that were faster for happy than for disgusted and fearful facial expressions, since the comparisons for happiness-disgust $[t(13)=4.51, p<.001$, $d=1.18]$ and happiness-fear $[t(13)=6.63, p<.001$, $d=1.60]$ were both significant. The main effect for scene was not significant $(p>.05)$.

The interaction between facial expression and scene was significant $\left[F(2.46,32.01)=4.74, p<.01, \eta_{\mathrm{p}}^{2}=\right.$ .27] (see Figure 2C). RTs were faster for disgusted facial expressions in disgusting than in fearful and happy scenes. RTs were also faster for fearful facial expressions in fearful scenes than in disgusting scenes, and RTs were faster for happy facial expressions in happy scenes than in disgusting and fearful scenes (see Table 2 for planned $t$-test comparisons).

\section{DISCUSSION}

The general increase in RTs indicates that the concurrent task competed for attentional resources. However, the effects of emotional scenes on RTs for facial expressions were not canceled. The pattern of error rates was similar to that shown in Experiment 1, in that disgust facial expressions were better recognized when paired with a disgustinducing scene. RTs were fastest for facial expressions in congruent scenes. Note that RTs were not significantly faster for fear facial expressions in fearful scenes than for those in happy scenes. This pattern was also observed in the previous experiments.

\section{GENERAL DISCUSSION}

In the present study, we have shown that the recognition of facial expressions is significantly affected by taskirrelevant emotional scenes. The effects of scenes were robust in the face of increasing task load. It is worth noting that it is unlikely that the physical properties of the stimuli explain the interactions between facial expression and scene. First, each scene served as a background for facial expressions of fear, disgust, and happiness, and is therefore congruent with some facial expressions and incongruent with others. Second, similar context effects have been observed in a word-naming task in which line drawings were used as context stimuli (de Houwer \& Hermans, 1994).

Two mechanisms may explain the observed effects, one possibly taking place during an early stage of processing (attentional selection/encoding), and the other at a late stage of processing (response selection).

First, the effects may be explained by early stages of face processing that differ as a function of the emotional context. Emotional information from scenes may be extracted rapidly (Calvo \& Lang, 2005) and may thus affect face processing rapidly (Righart \& de Gelder, 2006).
Previous work has shown that the N170 amplitude was larger for faces in fearful scene contexts than for those in neutral contexts, especially for fearful faces (Righart $\&$ de Gelder, 2006); this indicates that scenes are processed and combined with faces rapidly. Scene analysis may rely on the gist of the scene, in which information is extracted by fast perceptual analysis of global properties (Bar, 2004). A subcortical route, in which the amygdala is involved, may facilitate the processing of emotional information (de Gelder et al., 2006; LeDoux, 1996). It has been shown that the amygdala is critical for enhanced visual perception of emotional stimuli (Anderson \& Phelps, 2001; Vuilleumier, Richardson, Armony, Driver, \& Dolan, 2004). fMRI studies have shown correlations between amygdala and fusiform gyrus activity (Morris et al., 1998; Vuilleumier et al., 2004). An fMRI study has shown that amygdala activation for surprised facial expressions was increased when they were preceded by negative sentences, as compared with when they were preceded by positive sentences (Kim et al., 2004). In addition, left fusiform gyrus activation was increased (Kim et al., 2004), which suggests that negative emotional contexts may enhance face encoding (Righart \& de Gelder, 2006). Such effects may be explained by top-down modulation of attention or encoding, possibly by the prefrontal cortex (Kim et al., 2004). These enhanced responses in the fusiform gyrus may relate to face encoding or attention and may affect subsequent behavioral performance.

The results are also consistent with previous studies that have investigated attention for facial expressions that were flanked by congruent or incongruent facial expressions. The congruency effects of flankers were smaller for negative targets than for positive targets, perhaps because, for negative emotions, attention is more focal, which may reduce the impact of peripheral information (Fenske \& Eastwood, 2003; see also Rowe, Hirsh, \& Anderson, 2007).

A second mechanism that may explain the observed effects for contexts is related to response conflict. Congruency between facial expressions and emotional contexts may trigger faster reactions, because there is less response conflict in these conditions. The effects may then potentially be explained by mechanisms underlying the Stroop effect (Stroop, 1935). The face-scene pairs differ from the word-reading task used in the emotional Stroop (Algom, Chajut, \& Lev, 2004) and bar-orientation (Erthal et al., 2005) tasks, in that the dimensions of facial expression and scene overlap on emotion. These dimensions are associated with the same task-appropriate response, and as an effect, RTs may differ between congruent and incongruent pairs of faces and scenes (Algom et al., 2004). The observed errors were biased toward the context emotion, and there was no indication of speed-accuracy trade-offs. However, the absence of effects for fearful facial expressions in fearful scenes, as compared with those in happy scenes, and the small effects for negative schematic facial expressions in a previous study (Fenske \& Eastwood, 2003 ) introduce a challenge to an interpretation of response conflict based on the Stroop effect. Future studies need to investigate whether the effect still occurs when a task is used that does not generate response conflict, but 
in which the emotional facial expression and emotional context are task irrelevant.

Studies using Eriksen flanker tasks with negative and positive schematic facial expressions have shown that congruency effects for flanker faces were smaller for neutral or negative expressions than for positive expressions (Fenske \& Eastwood, 2003). In the experiments with short presentation times (1A and 2), disgust expressions were recognized significantly faster in disgust contexts than in happy contexts; but for fear faces, the difference between fear and happy context was not significant in any of the experiments. Our data are consistent with the finding by Fenske and Eastwood, in that we found congruency effects for happy faces in all comparisons.

To our knowledge, no behavioral experiment has reported about within-valence categorizations for the negative emotions of fear and disgust (but see recent fMRI data by Thielscher \& Pessoa, 2007). Categorization of negative emotions, such as fear and disgust, may be more difficult than categorization between positive and negative emotions, perhaps because attention is not only attracted to negative emotions more quickly (Blanchette, 2006; Hansen \& Hansen, 1988; Öhman, Flykt, \& Esteves, 2001; Öhman, Lundqvist, \& Esteves, 2001, but see also the arousal hypothesis by Schimmack \& Derryberry, 2005), but is also held by negative emotions (Fox, Russo, Bowles, \& Dutton, 2001). In addition, in most studies, more alternatives are used for negative emotions than for positive emotions (Calder, Young, Keane, \& Dean, 2000; Palermo \& Coltheart, 2004).

The observed interaction between facial expression and scene transcends the positive-negative valence dimension (e.g., positive facial expressions processed faster in positive contexts than in negative contexts, and vice versa). The categorization of facial expressions of disgust was faster in disgusting scenes than in fearful scenes, and categorization of fearful facial expressions was faster in fearful scenes than in disgusting scenes. Fast and efficient processing of specific facial expressions may help one to adapt rapidly to the environment (Ekman, 1992). Happy emotions are socially rewarding and may evoke affiliation (Keltner \& Kring, 1998). Fear is crucial to monitoring for potentially dangerous situations (LeDoux, 1996). Disgust keeps the individual away from a harmful object or contamination (Rozin \& Fallon, 1987).

The dissociation between fear and disgust processing adds to studies that have suggested that relatively separate neural systems process these emotions (see a review by Calder et al., 2001). Brain imaging studies have shown that the amygdala responds mainly to facial expressions of fear, whereas the insula-basal ganglia system responds mainly to disgust (Phillips et al., 1997), although others have found contrasting results (Anderson, Christoff, Panitz, De Rosa, \& Gabrieli, 2003; Fitzgerald, Angstadt, Jelsone, Nathan, \& Phan, 2006). Neuropsychological case studies have confirmed the importance of these brain regions for recognizing fear and disgust (Adolphs, Tranel, Damasio, \& Damasio, 1994; Sprengelmeyer et al., 1996), and fMRI data have shown the involvement of insula-basal ganglia in perceptual decisions for disgust, irrespective of stimulus features (Thielscher \& Pessoa, 2007). It would be interesting to study whether these brain regions are involved in processing faces in contexts and whether the activity is modified by congruent contexts. The relation between this brain activity - especially activity in regions of interest, like the amygdala and insula-basal ganglia system (Calder et al., 2001) - and behavioral responses for fear and disgust may be investigated in future studies by using parametrical designs, in which different facial expressions are accompanied by scenes of different emotional intensities.

\section{AUTHOR NOTE}

We are grateful to three anonymous reviewers for their useful comments and suggestions for improving the manuscript, and to Annemieke Vink for assistance in Experiment 1B. Correspondence concerning this article should be addressed to B. de Gelder, Universiteit van Tilburg, Warandelaan 2, Faculteit Sociale Wetenschappen, Departement Medische Psychologie en Neuropsychologie, Postbus 90153, 5000 LE Tilburg, The Netherlands (e-mail: b.degelder@uvt.nl).

\section{REFERENCES}

Adolphs, R. (2002). Recognizing emotion from facial expressions: Psychological and neurological mechanisms. Behavioral \& Cognitive Neuroscience Reviews, 1, 21-62.

Adolphs, R., Tranel, D., Damasio, H., \& Damasio, A. (1994). Impaired recognition of emotion in facial expressions following bilateral damage to the human amygdala. Nature, 372, 669-672.

Algom, D., Chajut, E., \& Lev, S. (2004). A rational look at the emotional Stroop phenomenon: A generic slowdown, not a Stroop effect. Journal of Experimental Psychology: General, 133, 323-338.

Anderson, A. K., Christoff, K., Panitz, D., De Rosa, E., \& GabriELI, J. D. E. (2003). Neural correlates of the automatic processing of threat facial signals. Journal of Neuroscience, 23, 5627-5633.

Anderson, A. K., \& Phelps, E. A. (2001). Lesions of the human amygdala impair enhanced perception of emotionally salient events. Nature, 411, 305-309.

Bar, M. (2004). Visual objects in context. Nature Reviews Neuroscience, 5, 617-629.

Barrett, L. F., Lindquist, K. A., \& Gendron, M. (2007). Language as context for the perception of emotion. Trends in Cognitive Sciences, 11, 327-332.

Biederman, I. (1972). Perceiving real-world scenes. Science, 177, $77-80$.

Blanchette, I. (2006). Snakes, spiders, guns, and syringes: How specific are evolutionary constraints on the detection of threatening stimuli? Quarterly Journal of Experimental Psychology, 59, 1484-1504.

Calder, A. J., Lawrence, A. D., \& Young, A. W. (2001). Neuropsychology of fear and loathing. Nature Reviews Neuroscience, 2, 352-363.

Calder, A. J., Young, A.W., Keane, J., \& Dean, M. (2000). Configural information in facial expression perception. Journal of Experimental Psychology: Human Perception \& Performance, 26, 527-551.

Calvo, M. G., \& LANG, P. J. (2005). Parafoveal semantic processing of emotional visual scenes. Journal of Experimental Psychology: Human Perception \& Performance, 31, 502-519.

Carroll, J. M., \& Russell, J. A. (1996). Do facial expressions signal specific emotions? Judging emotion from the face in context. Journal of Personality \& Social Psychology, 70, 205-218.

CoHen, J. (1988). Statistical power analysis for the behavioral sciences. Hillsdale, NJ: Erlbaum.

de Gelder, B., Meeren, H. K. M., Righart, R., Van Den Stock, J., Van De Riet, W. A. C., \& Tamietto, M. (2006). Beyond the face: Exploring rapid influences of context on face processing. Progress in Brain Research, 155, 37-48.

De Houwer, J., \& Hermans, D. (1994). Differences in affective processing of words and pictures. Cognition \& Emotion, 8, 1-20.

Eastwood, J. D., Smilek, D., \& Merikle, P. M. (2003). Negative facial expression captures attention and disrupts performance. Perception \& Psychophysics, 65, 352-358. 
Ekman, P. (1992). An argument for basic emotions. Cognition \& Emotion, 6, 169-200.

Erthal, F. S., De Oliveira, L., Mocaiber, I., Pereira, M. G., Machado-Pinheiro, W., Volchan, E., \& Pessoa, L. (2005). Loaddependent modulation of affective picture processing. Cognitive, Affective, \& Behavioral Neuroscience, 5, 388-395.

Fenske, M. J., \& Eastwood, J. D. (2003). Modulation of focused attention by faces expressing emotion: Evidence from flanker tasks. Emotion, 3, 327-343.

Fitzgerald, D. A., Angstadt, M., Jelsone, L. M., Nathan, P. J., \& Phan, K. L. (2006). Beyond threat: Amygdala reactivity across multiple expressions of facial affect. NeuroImage, 30, 1441-1448.

Fox, E., Russo, R., Bowles, R., \& Dutton, K. (2001). Do threatening stimuli draw or hold visual attention in subclinical anxiety? Journal of Experimental Psychology: General, 130, 681-700.

FriJdA, N. H., \& TCHERKASSOF, A. (1997). Facial expressions as modes of action readiness. In J. A. Russell \& J. M. Fernandez-Dols (Eds.), The psychology of facial expression (pp. 78-102). Cambridge: Cambridge University Press.

Hansen, C. H., \& Hansen, R. D. (1988). Finding the face in the crowd: An anger superiority effect. Journal of Personality \& Social Psychology, 54, 917-924.

Keltner, D., \& Kring, A. M. (1998). Emotion, social function, and psychopathology. Review of General Psychology, 2, 320-342.

Kim, H., Somerville, L. H., Johnstone, T., Polis, S., AlexanDER, A. L., Shin, L. M., \& Whalen, P. J. (2004). Contextual modulation of amygdala responsivity to surprised faces. Journal of Cognitive Neuroscience, 16, 1730-1745.

Lang, P. J., Bradley, M. M., \& CUthbert, B. N. (1999). International Affective Picture System (IAPS): Technical manual and affective ratings (Tech. Rep. C-1). Gainesville: University of Florida.

Lavie, N. (1995). Perceptual load as a necessary condition for selective attention. Journal of Experimental Psychology: Human Perception \& Performance, 21, 451-468.

LeDoux, J. E. (1996). The emotional brain: The mysterious underpinnings of emotional life. New York: Simon \& Schuster.

Loftus, G. R., Nelson, W. W., \& Kallman, H. J. (1983). Differential acquisition rates for different types of information from pictures. Quarterly Journal of Experimental Psychology, 35A, 187-198.

Lundevist, D., FlyKT, A., \& ÖHman, A. (1998). The Karolinska Directed Emotional Faces - KDEF [CD-ROM]. Stockholm: Karolinska Institutet, Department of Clinical Neuroscience, Psychology Section.

Meeren, H. K. M., van Heijnsbergen, C. C. R. J., \& De Gelder, B. (2005). Rapid perceptual integration of facial expression and emotional body language. Proceedings of the National Academy of Sciences, 102, 16518-16523.
Morris, J. S., Friston, K. J., Büchel, C., Frith, C. D., Young, A. W., Calder, A. J., \& Dolan, R. J. (1998). A neuromodulatory role for the human amygdala in processing emotional facial expressions. Brain, 121, 47-57.

Öhman, A., Flykt, A., \& Esteves, F. (2001). Emotion drives attention: Detecting the snake in the grass. Journal of Experimental Psychology: General, 130, 466-478.

Öhman, A., Lundqvist, D., \& Esteves, F. (2001). The face in the crowd revisited: A threat advantage with schematic stimuli. Journal of Personality \& Social Psychology, 80, 381-396.

Palermo, R., \& Coltheart, M. (2004). Photographs of facial expressions: Accuracy, response times, and ratings of intensity. Behavior Research Methods, Instruments, \& Computers, 36, 634-638.

Phillips, M. L., Young, A. W., Senior, C., Brammer, M., Andrew, C., CAlder, A. J., ET AL. (1997). A specific neural substrate for perceiving facial expressions of disgust. Nature, $\mathbf{3 8 9}, 495-498$

RighaRT, R., \& DE GELDER, B. (2006). Context influences early perceptual analysis of faces: An electrophysiological study. Cerebral Cortex, 16, 1249-1257.

Rowe, G., Hirsh, J. B., \& Anderson, A. K. (2007). Positive affect increases the breadth of attentional selection. Proceedings of the $\mathrm{Na}$ tional Academy of Sciences, 104, 383-388.

Rozin, P., \& Fallon, A. E. (1987). A perspective on disgust. Psychological Review, 94, 23-41.

Schimmack, U., \& Derryberry, D. (2005). Attentional interference effects of emotional pictures: Threat, negativity, or arousal? Emotion, 5, 55-66.

Sprengelmeyer, R., Young, A. W., Calder, A. J., Karnat, A., Lange, H., Homberg, V., ET AL. (1996). Loss of disgust: Perception of faces and emotions in Huntington's disease. Brain, 119, 1647-1665.

STROOP, J. R. (1935). Studies of interference in serial-verbal reactions. Journal of Experimental Psychology, 18, 643-662.

Tanaka-Matsumi, J., Attivissimo, D., Nelson, S., \& D’Urso, T. (1995). Context effects on the judgment of basic emotions in the face. Motivation \& Emotion, 19, 139-155.

Thielscher, A., \& PessoA, L. (2007). Neural correlates of perceptual choice and decision making during fear-disgust discrimination. Journal of Neuroscience, 27, 2908-2917.

Vuilleumier, P., Richardson, M. P., Armony, J. L., Driver, J., \& Dolan, R. J. (2004). Distant influences of amygdala lesion on visual cortical activation during emotional face processing. Nature Neuroscience, 7, 1271-1278.

(Manuscript received August 15, 2007; revision accepted for publication March 14, 2008.) 